\title{
Comparative Study of Different Image Resolution Enhancement Techniques in Wavelet Domain
}

\author{
Mrs.Smita.Y.Upadhye ${ }^{1}$, Mrs. Swapnali.B.Karole ${ }^{2}$ \\ Assistant Professor, Electronics Dept, DKTE's Textile \& Engineering Institute Ichalkaranji, India ${ }^{1}$ \\ Assistant Professor, EXTC Dept, DKTE's Textile \& Engineering Institute Ichalkaranji, India ${ }^{2}$
}

\begin{abstract}
Image resolution is an essential aspect of an image. The main objective of image enhancement is to process a given image so that the result image is more accurate than the original image for a specific application. It improves image features such as edges, boundaries which is more helpful for display and analysis. Here we propose an Image resolution enhancement techniques based on interpolation of the high frequency sub-band images. All high frequency sub-band images are obtained from wavelet zero padding-cycle spinning, discrete, stationary and Dual Tree Complex Wavelet Transform (DT-CWT). In this study, a comparison of three different image resolution enhancement techniques in wavelet domain is done. Each method is analyzed quantitatively and visually. Based on the analysis, the most efficient method is proposed. All the three techniques uses low resolution image as the input image and then wavelet transform is applied to decompose the input image into different high and low frequency sub-bands. Then these edges along with the input image are interpolated. Finally all these images are combined to generate a new resolution enhanced image by using inverse process.
\end{abstract}

Keywords: Interpolation, Wavelet Zero Padding, Discrete Wavelet Transform, Stationary Wavelet Transform, Dual Tree Complex Wavelet Transform

\section{INTRODUCTION}

Resolution is the most important quality aspect of images and videos. As the resolution of images is go on increasing the image becomes more clear and detailed. Image resolution enhancement is the process of manipulating an image so that resultant image is of good quality image. It actually increases clarity of images for human viewing. In recent year's in many of the image processing applications high resolution image is prime requirement such as, multiple description coding, medical imaging, video sequences and satellite imaging. A more frequently used technique for resolution enhancement is interpolation. An interpolation is the process of increasing the resolution of the image by selecting new pixel from surrounding one. This interpolation technique has been frequently used in many image processing applications such as super resolution [1-2].Facial reconstruction [3] and Multiple description coding [4]. Bicubic interpolation with the help of DWT and SWT is much better technique to obtain high resolution image[5]. Image resolution enhancement in wavelet domain is relatively a new research topic. Discrete Wavelet Transform (DWT) [6] is one of the recent wavelet transforms used in image processing. DWT divides an input image into four different subband images, like Low-Low (LL), Low-High (LH), High-Low (HL), and High-High (HH). Another recent wavelet transform which has been used in many image processing applications is Stationary Wavelet Transform (SWT) [7]. In short, SWT is similar to DWT but it does not use down-sampling due to this the subbands will have the same size as the input image.

In this work, we proposed three different image resolution enhancement techniques which gives high resolution image. In first technique wavelet zero padding and cycle spinning is applied to obtain high resolution image. In second technique DT-CWT is applied on input image to decompose input image into different sub-bands. These high frequency edges obtained from DT-CWT are interpolated using bicubic interpolation and these interpolated images are combined by using IDT-CWT to get high resolution image. Third technique utilizes DWT and SWT on input image to get high frequency subband image. These high frequency sub bands are interpolated using bicubic interpolation technique and interpolated images are combined by using the inverse DWT to achieve the high resolution image. All the three techniques are compared to get efficient technique 


\section{International Journal of Innovative Research in Electrical, Electronics, Instrumentation and Control Engineering}

Vol. 7, Issue 5, May 2019

\section{LITERATURE SURVEY}

Following are the literature review in image resolution enhancement done by some authors and their observations:

Robert G. Keys [8] proposed Bi-Cubic convolution technique which is applied on interpolation kernel. These Interpolation kernels are designed to maximize accuracy.Computing time of cubic convolution is less as compared to cubic splines.

Hasan Demirel et al [9] used DWT technique for interpolation of images. But the images obtained from DWT and IDWT technique are not sharper as compare to previous technique and they have low PSNR Value.

O.Harikrishna et al[10] introduced a new satellite image resolution enhancement method based on the interpolation of the high frequency sub band images obtained by Discrete Wavelet Transform (DWT) and the input image.

Hasan Demirel et al [11] In this paper Complex Wavelet Transform (CWT) is used for image processing. By applying CWT on an input image it gives two complex-valued low-frequency subband images and six complex valued highfrequency subband images. Then high frequency subband images and input image interpolated. All these real and imaginary valued images are used for the IDT-CWT operation. Quality and PSNR of the high resolution image is improved in this method.

G. N. S. Prasad, H. Khan, B.K. Muralidhar and T.S. Kiran [12] introduced a new method to improve the quality input image. In this technique both resolution and contrast features of images improved. This method uses DWT and SVD to increase the resolution and DWT and SWT is used to increase contrast. This process is based on the interpolation of HF sub band images obtained from DWT and input image. The edge details are enhanced by using SWT.

Darshana Mistry and Asim Banerjee [13] describes the fundamental information of DWT and implementation steps in MATLAB. In this technique initially image is filtered by using low pass filter and then filtered by high pass filter.By using DWT image is decomposed into multilevel sub-bands which include approximation details (LL sub band), horizontal detail (HL sub band), vertical (LH sub band) and diagonal details (HH sub band).

N. G. Kingsbury [14] introduced use of wavelets for multi-resolution image processing; he explained the implementation of filter-banks of Discrete Wavelet Transform (DWT) and extension for image processing and other multi-dimensional signals through separable filtering. He also explained the process of inversion of dwt. According to him complex wavelets can provide both good directional selectivity and shift invariance. But generation of a Complex Wavelet Transform (CWT) with proper reconstruction and good filter characteristics has proved difficult until recently.

T. H. Reeves and N. G. Kingsbury [15] this algorithm gives information about local edges from coarse scale coefficients, and determines the effect of these edges on the phases and magnitudes of finer level coefficients. Accurate prediction of coefficients has important benefits for resolution enhancement systems and image coding.

Kingsbury, N.G.[16] introduced a Dual-Tree Complex Wavelet Transform (DT CWT) with improved orthogonality and symmetry properties. The filters used in the two trees are time-reverse of each other, such as in analysis and reconstruction filters. This results into a transform, which uses shorter filters, and which is orthonormal after level 1, and which have symmetric sub-sampling structure. These two trees are closely matched. The key advantage of DT CWT is good directional selectivity and approximate shift-invariance in multiple dimensions.

\section{PROPOSED METHODS}

\section{A) Wavelet Zero Padding and Cycle Spinning (WZP-CS)}

In this method high resolution image is obtained from WZP method. In wavelet zero padding a wavelet transform is applied on low resolution image then a string of samples with zero values (zero matrix) is attached to the endpoint of the transformed image then by inverse of wavelet transform high resolution image is obtained [17].then by Using this high resolution image, $\mathrm{N}$ images of low resolution are obtained through spatial shifting and wavelet transforming and the high frequency components are discarded After that, again the WZP process is applied on all these low resolution images to obtain a number of images of high resolution. Final high resolution image is obtained by realignment and determining the average of these intermediate HR images. The generalised block diagram of this technique is as shown in figure1. 


\section{International Journal of Innovative Research in Electrical, Electronics, Instrumentation and Control Engineering}

Vol. 7, Issue 5, May 2019

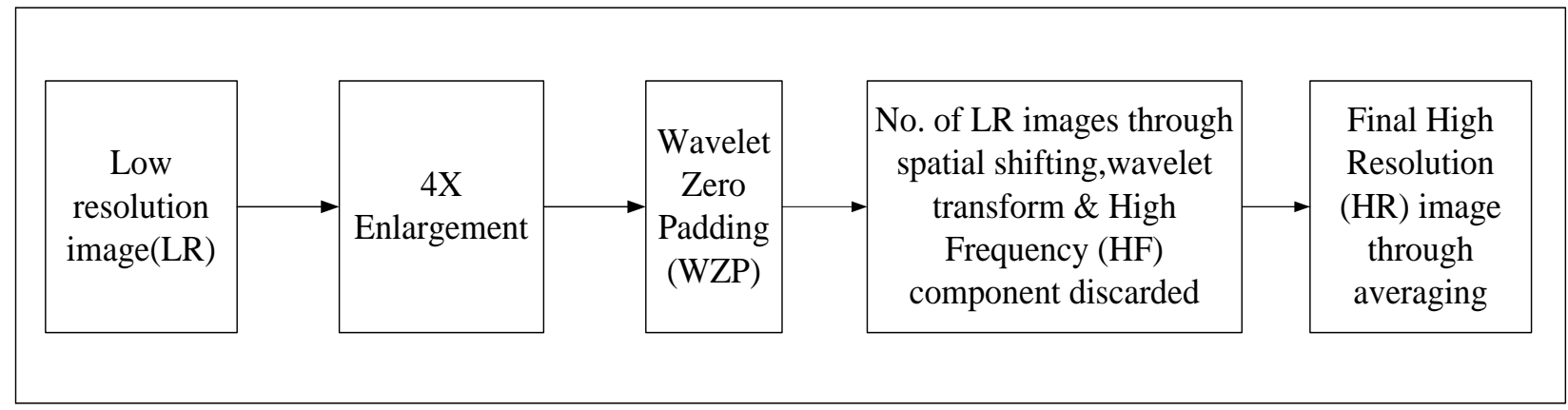

Figure1. Block diagram of WZP-CS

\section{B) Dual Tree-Complex Wavelet Transform (DT-CWT)}

DT-CWT is much better technique than the DWT technique. It was first introduced by N.G. Kingsbury to reduce the drawbacks of DWT because DT-CWT has better directional sensitivity and exhibits less redundancy. Basically DTCWT is a combination of two real discrete WT (DWT). The ordinary DWT is shift variant due to presence of decimation operation in the transform. And because of this, a small variation in the input signal will may produce a very different set of wavelet coefficients at the output. In this method, DT-CWT is used to preserve edges (i.e.) highfrequency components of the image.Figure 2 shows block diagram of DT-CWT technique. Following steps are used in this technique.

i) First DT-CWT is applied on input image that divides the input image into two low and six high frequency subband images.

ii) Then input image and high frequency sub-band images are interpolated by factor $\alpha / 2$ and $\alpha$ respectively.

iii) Finally, the interpolated high frequency subband images and input low resolution image are added by applying the inverse of DT-CWT to produce a high resolution image.

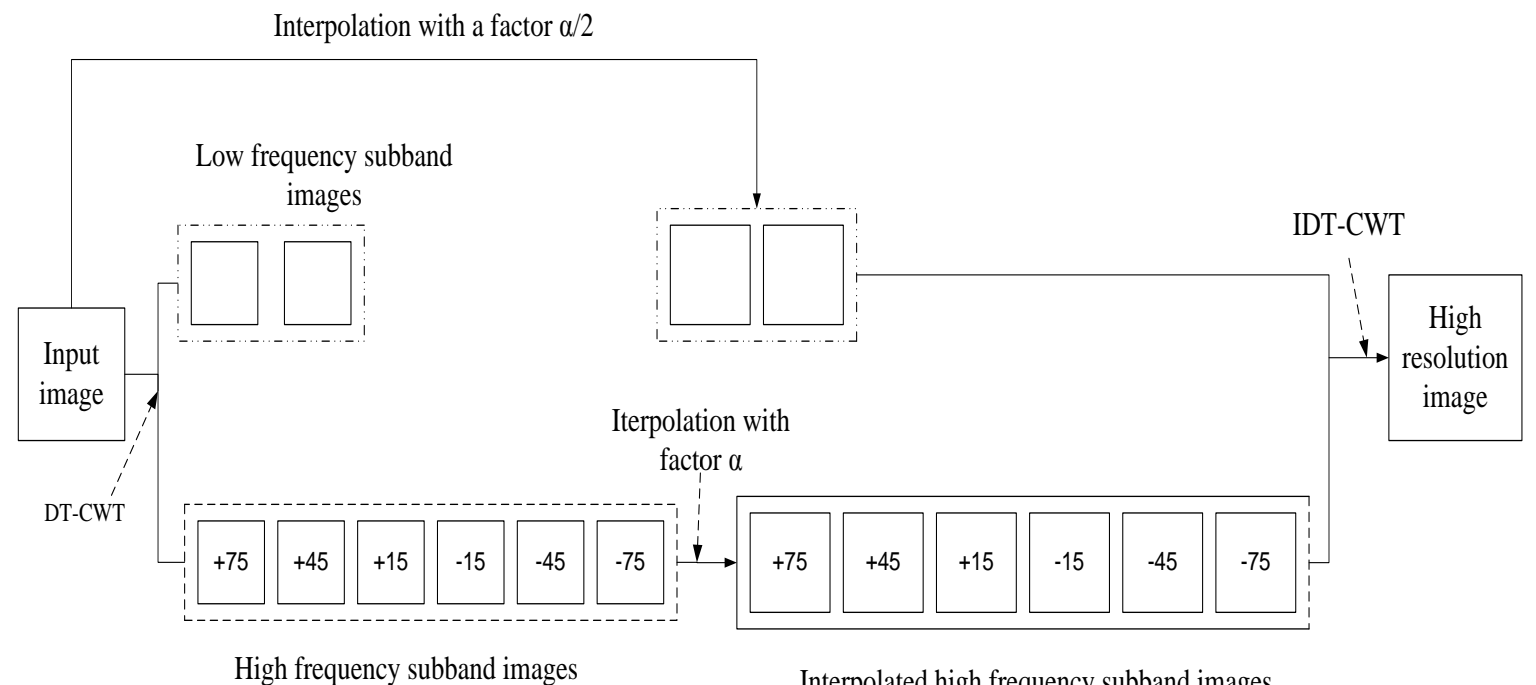

Figure2. Block diagram of DT-CWT

The real 2-D dual-tree DWT of an image is implemented using two critically-sampled 2-D DWTs in parallel form. Then for each pair of sub-bands sum and difference is calculated. The complex 2-D DT-DWT gives wavelets in six distinct directions. The complex 2-D dual-tree is created as four critically-sampled separable 2-D DWTs operating in parallel as shown in figure (3).This 2-D structure uses four trees for analysis and synthesis. The pair of conjugate filters applied to two dimensional images (x, y) can be expressed as:

$$
\left(h_{x}+j g_{x}\right)\left(h_{y}+j g_{y}\right)=\left(h_{x} h_{y}-g_{x} g_{y}\right)+j\left(h_{x} h_{y}+g_{x} g_{y}\right)
$$




\section{International Journal of Innovative Research in Electrical, Electronics, Instrumentation and Control Engineering}

Vol. 7, Issue 5, May 2019

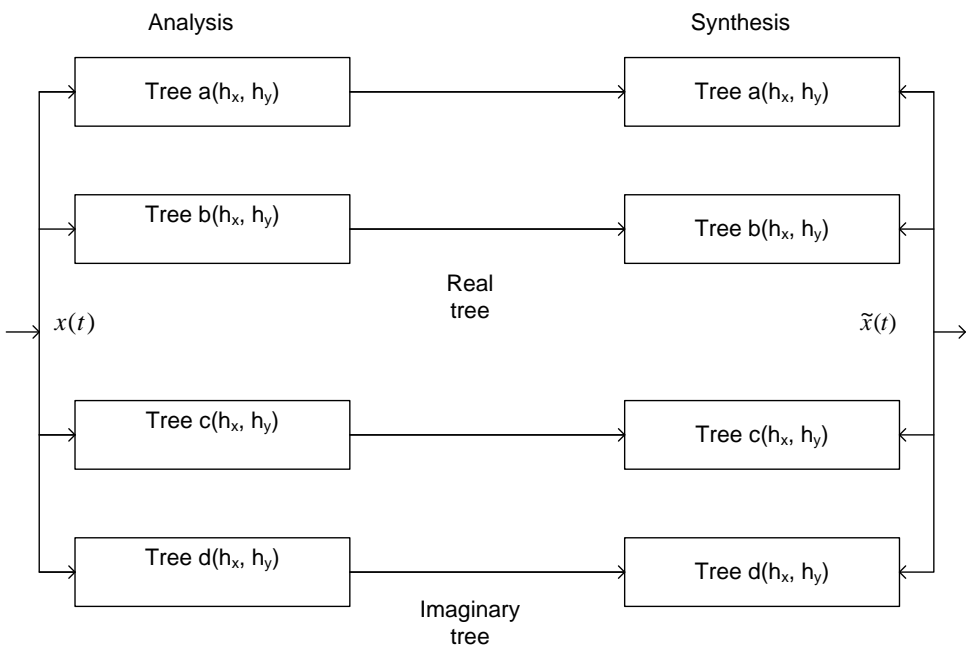

Figure3: Filter bank structure for 2-D DT-DWT

\section{C) DWT SWT technique}

The important part of an image is its edges and to increase the resolution of image preserving the edges Is essential as image resolution enhancement by interpolation method will result into loss of high frequency components which occurs because of smoothing caused by interpolation and thus, to improve the nature of the resolved image, it is necessary to retain all the edges/boundaries of the image. In practice, DWT is used for this purpose [17]. Hence Single level DWT is necessary to use to divide the input image into different sub-band images. Out of these sub-bands, the three sub-bands of high frequency namely: HL, LH and HH contain the edges. The decomposition of image after applying one level DWT and SWT are shown in figure4 and figure5 Low-Low (L.L) Low-High (LH.), High-Low(HL.), High-High (HH.) Frequency component. Block diagram of this technique is as shown in figure6

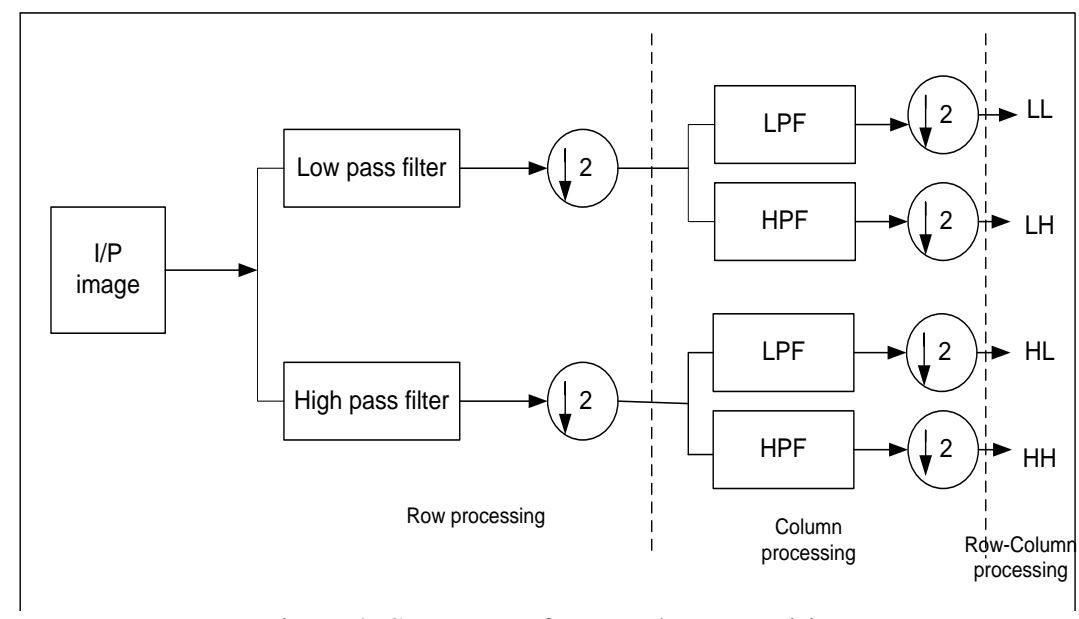

Figure4. Structure of DWT decomposition

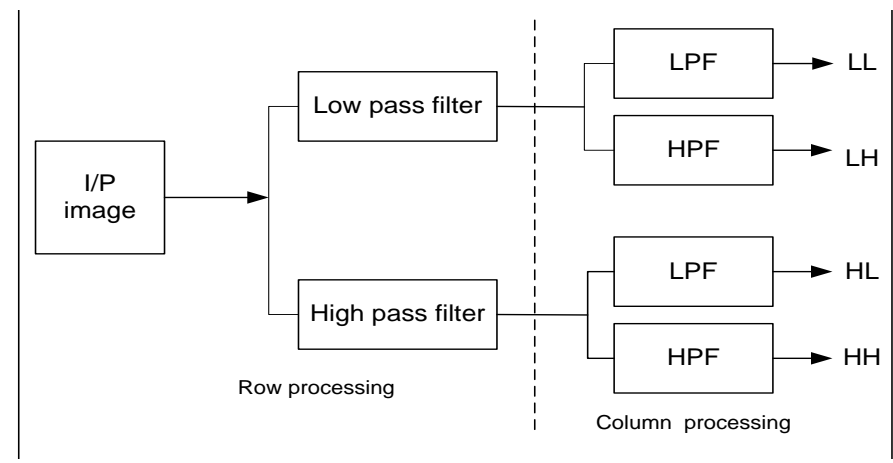

Figure5. Structure of SWT decomposition 


\section{International Journal of Innovative Research in Electrical, Electronics, Instrumentation and Control Engineering}

Vol. 7, Issue 5, May 2019

Steps involved in this technique are:-

i) Intially one level DWT is applied on low resolution image $(m \times n)$ to decompose an input image into four different sub bands as LL, LH, HL, and HH.Due to down sampling in DWT there is loss of data in subbands hence to minimize this loss SWT is used. That is SWT decomposes the input image using haar wavelet into four different sub bands i.e. LL, LH, HL, HH.

ii) The high frequency output i.e. LH, HL and HH of DWT technique is interpolated by using the bicubic interpolation technique with enlargement factor of 4. LH, HL and HH of SWT technique is interpolated by using the bicubic interpolation technique with enlargement factor of 2.

iii) Now all three high frequency components i.e LH, HH, HL of DWT and SWT technique have the same size hence they can be added together to get estimated high frequency sub-bands. These estimated high frequency sub-bands are again interpolated by factor $\alpha / 4$.

iv) Finally output of this bicubic interpolation and the original image which is interpolated by factor $\alpha / 2$ are combined by using inverse DWT (IDWT) to get the high resolution image.

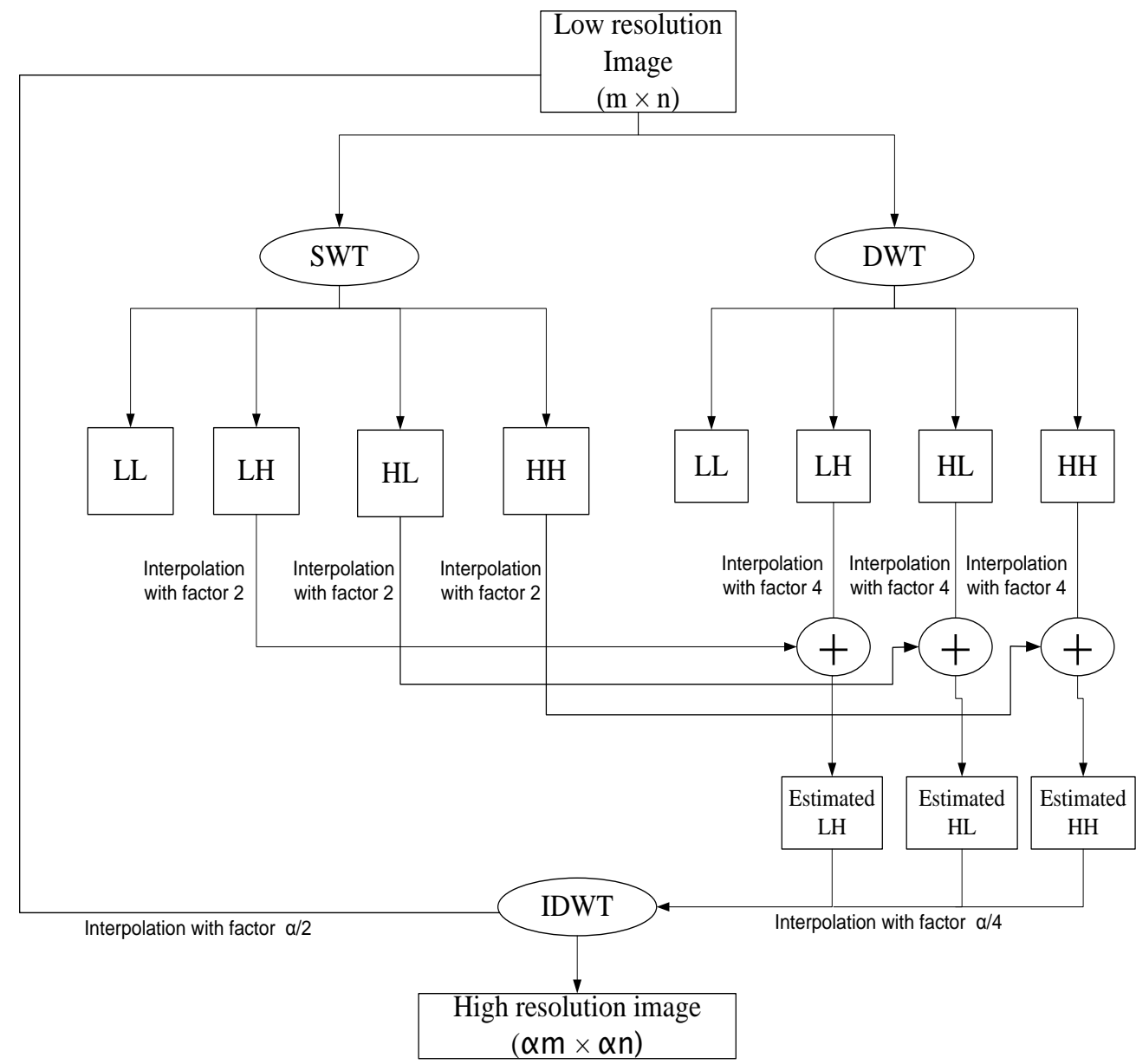

Figure6.Block diagram of DWT-SWT

IV.

PERFORMANCE PARAMETER

Here to check the superiority of high resolution image Peak Signal to Noise Ratio (PSNR) is calculated.Table1 compares PSNR results of three proposed technique. Different types of images such as standard matlab images, satellite images, and medical images are tested by this algorithm. The PSNR calculation of two images, one original and reconstructed image, describes how far two images are equal.

PSNR in DB given as

$\operatorname{PSNR}(\mathrm{DB})=10 \log _{10}\left(\mathrm{R}^{2} / \mathrm{MSE}\right)$

Where, $\mathrm{R}=$ peak value of the input image

$$
M S E=\frac{1}{M N} \sum_{i=0}^{m-1} \sum_{j=0}^{n-1}[I(i, j)-k(i, j)]^{2}
$$




\section{International Journal of Innovative Research in Electrical, Electronics, Instrumentation and Control Engineering}

Vol. 7, Issue 5, May 2019

$\mathrm{M} \& \mathrm{~N}$ are the size of the images, $\mathrm{M}=$ no. of rows $\mathrm{N}=$ no. of columns and are the matrix element of the reconstructed image and the original image at (i,j)th pixel respectively. The result in table 1 shows that proposed method 3 is superior than method1 \& method2.

\section{RESULTS AND DISCUSSION}

Figure7, Figure8 and Figure9 show the low resolution input \& high resolution output images of method1, 2, and 3 respectively. Table 1 compares the PSNR performance of all WZP-CS, DT-CWT \& DWT, and SWT technique. The result in table1 indicates that the DWT, SWT technique gives much better result as compared to WZP-CS and DT-CWT technique.

Table1 .Observation Table

\begin{tabular}{|l|l|l|l|}
\hline & \multicolumn{2}{|l|}{ PSNR(dB) } \\
\hline Method/Image & Proposed Method1 & Proposed Method2 & Proposed Method3 \\
\hline X-Ray image & 37.80 & 26.40 & 44.65 \\
\hline Satellite image & 32.96 & 28.63 & 38.74 \\
\hline Standard Baboon image & 32.23 & 26.87 & 47.66 \\
\hline
\end{tabular}

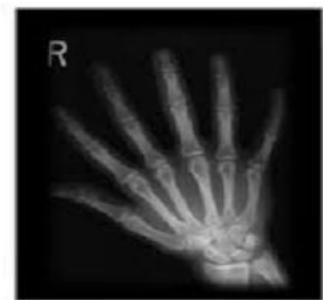

(a)

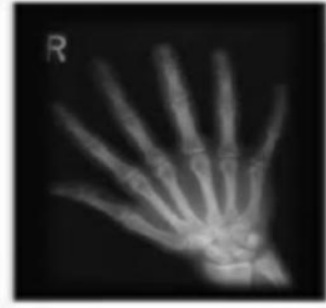

(c)

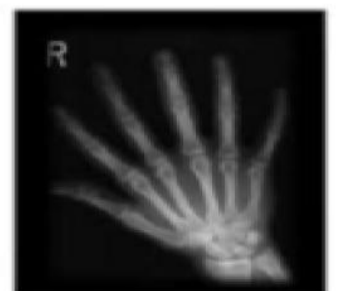

(b)

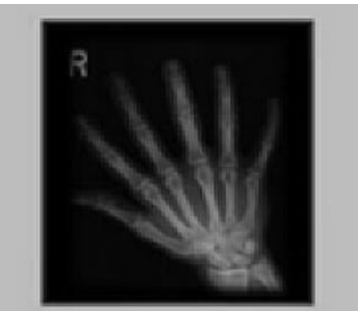

(d)

Figure7. (a) original X-ray image (b) WZP-CS (c) DT-CWT (d) DWT-SWT technique

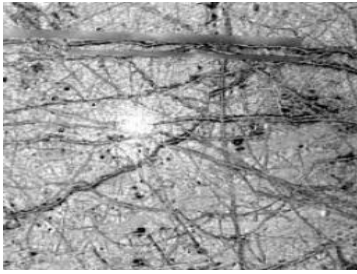

(a)

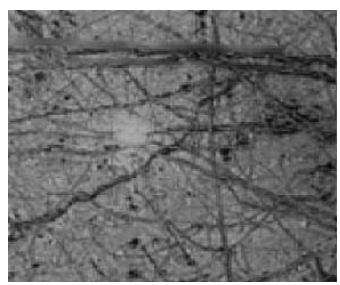

(c)

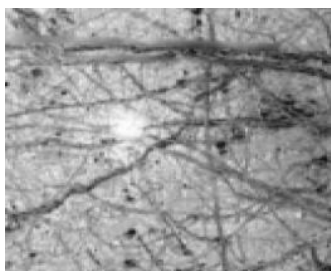

(b)

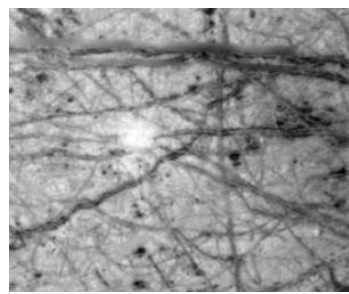

(d)

Figure8. (a) Original Satellite image (b) WZP-CS (c) DT-CWT (d) DWT-SWT technique 


\section{International Journal of Innovative Research in Electrical, Electronics, Instrumentation and Control Engineering}

Vol. 7, Issue 5, May 2019

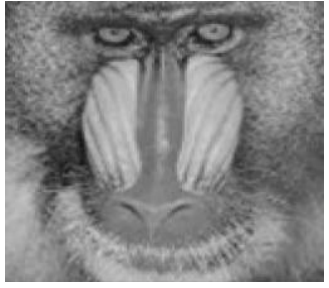

(a)

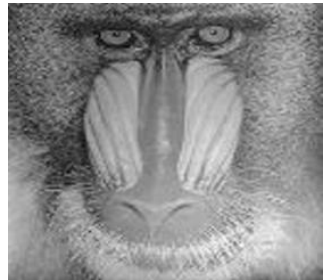

(c)

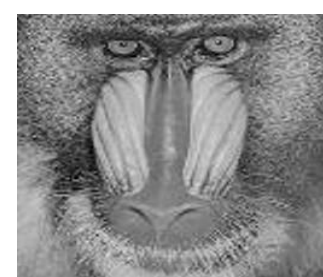

(b)

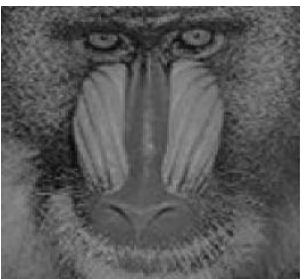

(d)

Figure9. (a) Original Baboon image (b) WZP-CS (c) DT-CWT (d) DWT-SWT technique

VI.

CONCLUSION

This paper analyzes three different methods to obtain high resolution images from low resolution images. All these three image resolution enhancement techniques WZP-CS, DT-CWT, DWT-SWT have been tested on Lena, Baboon, Elaine and Pepper image. This technique is also applied to 4 satellite images and 5 medical images. Actually this work involves decomposition of low resolution image by using DWT which gives high frequency subbands. High frequency subbands are interpolated to increase their size. DWT loses some information due to interpolation process. This loss of information is corrected by SWT. Interpolated high frequency subbands and interpolated input image is combined by using inverse DWT(IDWT) to achieve the high resolution image. The performance of these three methods has been compared on the basis of PSNR where, PSNR result shows that maximum PSNR value using DWT, SWT is 47.66.

\section{REFERENCES}

[1]. L. Yi-bo, X. Hong, and Z. Sen-yue, "The wrinkle generation method for facial reconstruction based on extraction of partition wrinkle line features and fractal interpolation," in Proc. 4th Int. Conf. Image Graph., Aug. 22-24, 2007,pp. 933-937.

[2]. H. Demirel, G. Anbarjafari, and S. Izadpanahi, "Improved motion based localized super resolution technique using discrete wavelet transform for low resolution video enhancement," in Proc. 17th Eur. Signal Process. Conf., Glasgow, Scotland, Aug. 2009, pp. 1097-1101.

[3]. Y. Rener, J. Wei, and C. Ken, "Downsample-based multiple description coding and post-processing of decoding," in Proc. 27th Chinese Control Conf., Jul. 16-18, 2008, pp. 253-256.

[4]. C. B. Atkins, C. A. Bouman, and J. P. Allebach, "Optimal image scaling using pixel classification," in Proc. Int. Conf. Image Process., Oct. 710, 2001, vol. 3, pp. 864-867.

[5]. Hasan Demirel and Gholamreza Anbarjafari "IMAGE Resolution Enhancement by Using Discrete and Stationary Wavelet Decomposition", IEEE TRANSACTIONS ON IMAGE PROCESSING, VOL. 20, NO. 5, MAY 2011

[6]. S. Mallat, A Wavelet Tour of Signal Processing, 2nd ed. New York: Academic, 1999.

[7]. J. E. Fowler, "The redundant discrete wavelet transform and additive noise," Mississippi State ERC, Mississippi State University, Tech. Rep. MSSU-COE-ERC-04-04, Mar. 2004.

[8]. Robert G. Keys," cubic convolution interpolation for digital image processing”, IEEE transactions on acoustics, speech and signal processing, vol-29, no.6,December 1981.

[9]. Hasan Demirel and Gholamreza Anbarjafari,"Discrete Wavelet Transform-Based Satellite Image Resolution Enhancement" IEEE Trans. geoscience and remote sensing letters,vol.49,no.6, June 2011.

[10]. O.Harikrishna, A.Maheshwari," Satellite Image Resolution Enhancement using DWT Technique”, International Journal of Soft Computing and Engineering(IJSCE) ISSN: 2231-2307, Volume-2, Issue-5, November 2012,pp.274-278

[11]. H. Demirel and G. Anbarjafari, "Satellite image resolution enhancement using complex wavelet transform," IEEE Geoscience and Remote Sensing Letter, vol. 7, no. 1, pp. 123-126, Jan. 2010.

[12]. G. N. S. Prasad, H. Khan, B.K. Muralidhar and T.S. Kiran, "Image Enhancement Using Wavelet Transforms and SVD", International Journal of Engineering Science and Technology (IJEST), vol. 4, no.03, pp. 1080-1087, 2012.

[13]. K. R. Castleman, "Digital Image Processing", 1st edition, NJ- Prentice Hall, 1996.

[14]. N. G. Kingsbury, "Image processing with complex wavelets,” Philos. Trans. R. Soc. London A, Math. Phys. Sci., vol. 357, no. 1760, pp. 25432560, Sep. 1999.

[15]. T. H. Reeves and N. G. Kingsbury, "Prediction of coefficients from coarse to fine scales in the complex wavelet transform," in Proc. IEEE ICASSP, Jun. 5-9, 2000, vol. 1, pp. 508-511.

[16]. Kingsbury, N.G.(2000) "A dual-tree complex wavelet transform with improved orthogonality and symmetry properties. Proc. IEEE Conf. on Image Processing, Vancouver, September 11-13, 2000, (paper 1429). 